\title{
ARTIGOS
}

\section{O VÍRUS DA HEPATITE B NA DOENÇA HEPÁTICA ALCOÓLICA: AVALIAÇÃO CLÍNICA E BIOQUÍMICA}

\author{
Celina M.C. Lacet e Edna Strauss
}

\begin{abstract}
Foram estudados prospectiva e sequencialmente 107 pacientes com doença hepática alcoólica (DHA) crônica, sendo 83 casos de cirrose e 24 de hepatite alcoólica. Além do etilismo, ingestão minima de 70 gramas de etanol puro ao dia por periodo superior a sete anos, todos apresentaram disfunção hepatocelular. De acordo com o perfil sorológico para o VHB os pacientes foram alocados em quatro grupos a saber: grupo I infectados: AgHBs e anti-HBC positivos; grupo II imunes: anti-HBs e anti-HBc positivos; grupo III sem marcadores do VHB: AgHBs, anti-HBc e anti-HBs negativos; grupo IV anti-HBc positivo isoladamente. A prevalência de infecção pelo VHB na DHA foi alta: 42,06\% apesar dos indices pouco elevados de imunidade: $26,17 \%$ sugerindo que na DHA ocorre maior exposição ao VHB com resposta imunológica deficiente. $A$ análise dos parâmetros clínico-bioquímicos, quando considerados isoladamente, não mostrou diferenças estatisticamente significantes entre os grupos I, II, e III, entretanto a classificação de Child/Campbell, discriminou o grupo infectado, onde houve predominio da classe $C$, de pior prognóstico.
\end{abstract}

Palavras Chaves: Doença hepática alcoólica. Virus da hepatite B. AgHBs. Avaliação clínico-bioquímica.

Na gênese da doença hepática crônica o álcool e a infeç̧ão pelo vírus da hepatite $B$ (VHB) são os - fatores etiológicos mais importantes, razão pela qual o estudo desta associação tem despertado interesse crescente nos últimos anos.

Uma possível relação entre doença hepática alcoólica (DHA) e VHB, foi aventada inicialmente no trabalho de Pettigrew e colaboradores ${ }^{34}$, após o qual alguns estudos demosntraram que o anti-HBs se apresentava em percentuais de positividade significativamente maiores na DHA que em seus grupos controles ${ }^{4} 283640$ resultados não comprovados por outros autores 51113233041 .

A determinação do AgHBs, tando isolado como associado ao anti-HBc, realizada na DHA, demonstrou maiores índices de positividade quando comparada a grupos controles, em alguns estudos 5 1230 , porém esta diferença não foi significativa em outros trabalhos ${ }^{4131936}$.

São escassos os estudos que procuram caracterizar, através de parâmetros clínicos e/ou bioquímicos, esta associação, sendo discordantes

Trabalho da Clínica de Gastroenterologia do Hospital Heliópolis, SP.

Endereço para correspondência: Prof ${ }^{\text {a }}$ Edna Strauss. R. Nebraska 251/13, 04560-010 São Paulo SP, Brasil.

Recebido para publicação em 04/06/93. os seus resultados 2228293035 . Objetivamos neste estudo, verificar em nosso meio, a prevalência da infeç̧ão pelo VHB na DHA e discriminar, através de parâmetros clínicos e bioquímicos, os grupos de DHA com e sem marcadores do VHB.

\section{MATERIAL E MÉTODOS}

Foram estudados prospectiva e seqüencialmente 107 portadores de doença hepática alcoólica, sendo 83 com cirrose hepática e 24 com hepatite alcoólica, todos internados na Clínica de Gastroenterologia do Hospital Heliópolis, no período de 1983 a 1985.

Os critérios de inclusão no trabalho foram: 1 ingestão mínima de 70 gramas de etanol por dia por período superior a sete anos, associado a evidências clínicas e bioquímicas de disfunção hepatocelular e; 2 - determinação do perfil sorológico para VHB (AgHBs, anti-HBc e anti-HBs - por Elisa/Abbott).

Foi realizada biópsia hepática, com confirmação histopatológica de DHA, em 75 pacientes, sendo o diagnóstico apenas macroscópico, através de laparoscopia, em outros 12 . Em 20 casos, cujas alterações da coagulação sangüínea contraindicavam até mesmo a exploração laparoscópica, o diagnóstico baseou-se na associação dos seguintes dados clínicos e laboratoriais, segundo os critérios de Garcia-Tsao 
Celina MC, Lacet e Edna Strauss. $O$ vírus da hepatite $B$ na doença hepática alcoólica: avaliação clínica e bioquímica. Revista da Sociedade Brasileira de Medicina Tropical 26:201-209, out-dez, 1993.

e cols ${ }^{17}$ : icterícia, hepatomegalia, ascite, aranhas vasculares, eritema palmar, hipoalbuminemia, hipergamaglobulinemia, alargamento do tempo de protrombina e varizes de esôfago.

Os 107 pacientes foram distribuídos em quatro grupos, de acordo com os resultados da pesquisa sorológica para VHB, a saber:

grupo I - pacientes AgHBs e anti-HBc positivos (infectados);

grupo II - pacientes anti-HBs e anti-HBc positivos (imunes);

grupo III - pacientes AgHBs, anti $\mathrm{HBc}$ e antiHBs negativos;

grupo IV - pacientes anti-HBc positivos, isoladamente.

Foram pesquisados também dados epidemiológicos considerados importantes para definir contatos prévios com VHB, tais como: história de hepatite, manipulações e contatos de risco para o VHB, transfusão de sangue e/ou hemocomponentes.

A sintomatologia pesquisada incluía queixas de astenia, icterícia, colúria, além de manifestações de descompensação hepática como: hematêmese, melena, ascite, edemas e alterações neuropsíquicas.

No exame físico foram valorizados os achados de desnutrição, atrofia muscular, anemia, icterícia, aranhas vasculares, eritema palmar, ginecomastia, circulação colateral, ascite, edema, fígado e baço palpáveis além de alterações neuropsíquicas.

Com relação ao estudo bioquímico foram determinadas as atividades enzimáticas de alanina aminotranferase (ALT), aspartato aminotranferase (AST), fosfatase alcalina (FA), gamaglutamiltranspeptidase (CGT), as dosagens de bilirrubinas totais e frações em $\mathrm{mg} \%$ e determinação eletroforética das proteínas sangüíneas em $\mathrm{g} \%$.

As alterações da coagulação sangüinea foram avaliadas através dos seguintes fatores: tempo de protrombina, plaquetas, fator $\mathrm{V}$ e fibrinogênio.

A classificação de Child-Campbell modificada ${ }^{9}$ foi aplicada sistematicamente em todos os pacientes. Como resultado da somatória dos pontos atribuídos a dados clínicos e bioquímicos, os pacientes foram classificados nos grupos A, B ou C.

Análise estatística: O teste de qui-quadrado de Pearson foi aplicado para as variáveis qualitativas ou classificatórias ${ }^{16}$. Na classificação de Child/ Campbell foi utilizada estimativa por máxima verossimilhança no ajuste de um modelo log-linear, prosseguindo-se com análise de variância e de contraste ${ }^{6}$. Análise multivariada foi utilizada nas variáveis de resposta quantitativa ${ }^{1}$. O nível de significância adotado foi de 0,05 .

\section{RESULTADOS}

A idade média dos pacientes foi de $46,06 \mathrm{com}$ desvio padrão de 10,02 , mínima de 26 anos e máxima de 74 anos. Houve nítida predominância do sexo masculino com $94(87,95 \%)$ casos e apenas 13 $(12,15 \%)$ casos do sexo feminino. Quanto à cor, houve predomínio dos brancos com $93(86,92 \%)$ casos, seguida dos pardos com $11(10,28 \%)$ casos, amarelos $2(1,87 \%)$ casos e negro apenas $1(0,93 \%)$ caso.

Os resultados de prevalência dos marcadores VHB na DHA, distribuídos nos quatro grupos, constam da Tabela 1. O grupo IV não foi incluído nas análises estatísticas devido ao pequeno número de casos.

Tabela $1-$ Agrupamento dos pacientes com DHA
conforme os resultados dosmarcadores para
o VHB.

\begin{tabular}{rrrrrr}
\hline \multirow{2}{*}{ Grupo } & AgHBs & Anti-HBc & Anti-HBs & \multicolumn{2}{c}{ Casos } \\
\cline { 3 - 6 } & & & \multicolumn{1}{c}{$\mathrm{n}^{\mathbf{0}}$} & $\%$ \\
\hline I & + & + & - & 17 & 15,89 \\
II & - & + & + & 28 & 26,17 \\
III & - & - & - & 55 & 51,40 \\
IV & - & + & - & 7 & 6,54 \\
\hline \multirow{2}{*}{ Total } & & & & 107 & 100,00 \\
\hline
\end{tabular}

O tempo de etilismo, a quantidade de etanol (em gramas) ingerida por dia e o tempo de suspensão da bebida alcoólica expressa em meses, sofreram análises comparativas, não sendo detectada qualquer diferença entre os grupos (Tabela 2).

Dados epidemiológicos considerados como fatores de risco para o contágio com o VHB foram igualmente analisados (Tabela 3) não se mostrando discriminatórios entre os pacientes infectados, imunes, ou sem prévio contato com o VHB.

$O$ resultado da análise de parâmetros clínicos como astenia, icterícia, colúria, hematêmese, melena, alterações neuropsíquicas, ascite e edema (Tabela 4), além de sinais ao exame físico de: 
Celina MC, Lacet e Edna Strauss. O vírus da hepatite B na doença hepática alcoólica: avaliação clínica $e$ bioquímica. Revista da Sociedade Brasileira de Medicina Tropical 26:201-209, out-dez, 1993.

Tabela 2 - Tempo de etilismo e quantidade diária de ingestão etílica nos três grupos de estudo.

\begin{tabular}{cccccrcc}
\hline & Grupo & $\mathrm{n}^{0}$ casos & Média & DP & Min & Max & "p" \\
\hline Tempo & I & 17 & 16,82 & 6,93 & 7 & 30 & \\
etilismo (anos) & II & 28 & 21,67 & 7,39 & 10 & 40 & 0,085 \\
& III & 55 & 20,83 & 8,48 & 8 & 45 & \\
Quantidade & I & 17 & 189,29 & 130,41 & 80 & 500 & \\
(d/g) & II & 28 & 209,75 & 117,70 & 70 & 515 & 0,627 \\
& III & 55 & 187,07 & 97,59 & 70 & 500 & \\
\hline
\end{tabular}

"p" = probabilidade estatística; DP = desvio padrão.

Obs.: o "p" foi aplicado ao logarítimo das variáveis.

Tabela 3 - Dados epidemiológicos relacionados à infeç̧ão pelo VHB.

\begin{tabular}{|c|c|c|c|c|c|c|}
\hline \multirow{2}{*}{ Grupo } & \multicolumn{2}{|c|}{$\begin{array}{l}\text { História } \\
\text { de hepatite }\end{array}$} & \multicolumn{2}{|c|}{$\begin{array}{l}\text { Manipulações } \\
\text { e contatos }\end{array}$} & \multicolumn{2}{|c|}{$\begin{array}{l}\text { Transfusões de sangue } \\
\text { e hemocomponentes }\end{array}$} \\
\hline & $n^{0}$ & $\%$ & $n^{0}$ & $\%$ & $n^{0}$ & $\%$ \\
\hline I & 1 & 5,88 & 3 & 17,65 & 2 & 11,76 \\
\hline II & 1 & 3,57 & 10 & 35,71 & 10 & 35,71 \\
\hline III & 6 & 10,91 & 19 & 34,55 & 14 & 25,45 \\
\hline$X^{2}$ & & & \multicolumn{2}{|c|}{1,95} & \multicolumn{2}{|c|}{3,17} \\
\hline "p" & & & \multicolumn{2}{|c|}{0,377} & \multicolumn{2}{|c|}{0,204} \\
\hline
\end{tabular}

$\mathrm{X}^{2}=$ qui-quadrado; "p" = probabilidade estatística

Tabela 4 - Sintomas de doença hepática referidos nos três grupos de estudo.

\begin{tabular}{|c|c|c|c|c|c|c|c|c|}
\hline \multirow[t]{2}{*}{ Grupo } & \multicolumn{2}{|c|}{ I } & \multicolumn{2}{|c|}{ II } & \multicolumn{2}{|c|}{ III } & \multirow[b]{2}{*}{$\mathrm{X}^{2}$} & \multirow[b]{2}{*}{ "p" } \\
\hline & $n^{\circ}$ & $\%$ & $n^{0}$ & $\%$ & $n^{0}$ & $\%$ & & \\
\hline Astenia & 3 & 17,65 & 3 & 10,71 & 9 & 16,36 & $\cdots$ & 0,799 \\
\hline Ictericia & 11 & 64,71 & 14 & 50,00 & 27 & 49,09 & 1,33 & 0,514 \\
\hline Colúria & 8 & 47,06 & 4 & 14,29 & 14 & 25,45 & 1,95 & 0,377 \\
\hline Hematêmese & 6 & 35,29 & 9 & 32,14 & 10 & 18,78 & 3,08 & 0,214 \\
\hline Melena & 6 & 35,29 & 10 & 35,71 & 13 & 23,64 & 1,71 & 0,426 \\
\hline Alterações neuropsíquicas & 1 & 5,88 & 8 & 28,57 & 3 & 5,45 & - & 0,012 \\
\hline Edema & 8 & 47,06 & 12 & 42,86 & 14 & 25,45 & 4,06 & 0,131 \\
\hline Ascite & 9 & 62,94 & 17 & 60,71 & 24 & 43,64 & 1,71 & 0,426 \\
\hline
\end{tabular}

$\mathrm{X}^{2}=$ qui-quadrado; "p" = probabilidade estatística 
Celina MC, Lacet e Edna Strauss. $O$ vírus da hepatite B na doença hepática alcoólica: avaliaçâo clínica $e$ bioquímica. Revista da Sociedade Brasileira de Medicina Tropical 26:201-209, out-dez, 1993.

desnutrição, atrofia muscular, anemia, icterícia, aranhas vasculares, circulação colateral, ginecomastia, ascite e edema (Tabela 5) não apresentaram significância estatística na separação dos grupos.

A determinação enzimática da AST, ALT, FA e gama GT (Tabela 6); níveis de bilirrubinas totais e frações (Tabela 7); eletroforese de proteínas (Tabela 8) e alterações de coagulação sangüínea (Tabela 9) foram analisados, sem que se conseguisse qualquer diferença significativa entre os grupos que pudesse servir como marcador da associação entre DHA e VHB.

Finalmente, ao realizarmos a comparação dos grupos utilizando a classificação de Child/Campbell modificada foi possível demonstrar que a classe $\mathrm{C}$ de Child/Campbell foi mais freqüente no grupo infectado, quando comparado ao grupo imune e sem marcadores do VHB (Tabela 10).

Tabela 5 - Sinais de doença hepática encontrados ao exame físico.

\begin{tabular}{|c|c|c|c|c|c|c|c|c|}
\hline \multirow[t]{2}{*}{ Grupo } & \multicolumn{2}{|c|}{ I } & \multicolumn{2}{|c|}{ II } & \multicolumn{2}{|c|}{ III } & \multirow[b]{2}{*}{$X^{2}$} & \multirow[b]{2}{*}{ "p" } \\
\hline & $\mathrm{n}^{\mathrm{o}}$ & $\%$ & $n^{\circ}$ & $\%$ & $n^{o}$ & $\%$ & & \\
\hline Desnutrição & 7 & 41,18 & 11 & 39,28 & 18 & 32,73 & 0,59 & 0,747 \\
\hline Atrofia muscular & 7 & 41,18 & 10 & 35,71 & 15 & 27,27 & 1,40 & 0,479 \\
\hline Anemia & 6 & 35,29 & 12 & 42,86 & 24 & 43,64 & 0,38 & 0,826 \\
\hline Icterícia & 11 & 64,71 & 18 & 64,29 & 33 & 60,00 & 0,21 & 0,901 \\
\hline Aranhas vasculares & 9 & 52,94 & 17 & 60,71 & 30 & 54,55 & 0,36 & 0,834 \\
\hline Eritema palmar & 3 & 17,65 & 11 & 39,29 & 20 & 36,36 & 2,51 & 0,285 \\
\hline Circulação colateral & 4 & 23,53 & 8 & 28,57 & 8 & 14,55 & 2,44 & 0,295 \\
\hline Ginecomastia & 1 & 5,88 & 4 & 14,28 & 4 & 7,27 & -- & 0,626 \\
\hline Hepatomegalia & 16 & 94,12 & 23 & 82,14 & 50 & 90,91 & - & 0,453 \\
\hline Esplenomegalia & 5 & 29,41 & 8 & 26,57 & 13 & 23,64 & 0,36 & 0,836 \\
\hline Ascite & 11 & 64,71 & 16 & 57,14 & 27 & 49,09 & 1,43 & 0,489 \\
\hline Edema & 7 & 41,18 & 11 & 39,29 & 19 & 34,55 & 0,33 & 0,847 \\
\hline
\end{tabular}

$\mathrm{X}^{2}=$ qui-quadrado; $" \mathrm{p} "=$ probabilidade estatística

Tabela 6 - Enzimas hepáticas nos três grupos de estudo*

\begin{tabular}{|c|c|c|c|c|c|c|c|}
\hline & Grupo & $\mathrm{N}$ & Média & $\mathrm{DP}$ & Min & $\operatorname{Max}$ & "p" \\
\hline \multirow[t]{3}{*}{ AST/VMN } & I & 17 & 3,57 & 3,42 & 0,92 & 11,60 & \multirow{3}{*}{0,477} \\
\hline & II & 28 & 2,34 & 1,20 & 0,64 & 5,03 & \\
\hline & III & 55 & 2,41 & 2,10 & 0,28 & 8,65 & \\
\hline \multirow[t]{3}{*}{ ALT/VMN } & I & 17 & 2,40 & 4,38 & 0,63 & 19,00 & \multirow{3}{*}{0,477} \\
\hline & II & 28 & 1,29 & 0,68 & 0,38 & 3,78 & \\
\hline & III & 55 & 1,30 & 1,11 & 0,05 & 5,44 & \\
\hline \multirow[t]{3}{*}{ FA/VMN } & I & 16 & 2,21 & 1,66 & 0,57 & 7,78 & \multirow{3}{*}{0,171} \\
\hline & II & 28 & 1,88 & 1,45 & 0,59 & 7,09 & \\
\hline & III & 52 & 1,54 & 0,69 & 0,17 & 3,07 & \\
\hline \multirow[t]{3}{*}{ Gama GT/VMN } & I & 17 & 5,16 & 5,97 & 0,97 & 24,84 & \multirow{3}{*}{0,060} \\
\hline & II & 28 & 4,07 & 4,27 & 0,70 & 16,54 & \\
\hline & III & 55 & 7,58 & 7,55 & 0,43 & 32,90 & \\
\hline
\end{tabular}

* Valores transformados em $n^{0}$ de vezes o valor máximo normal (VMN)

"p" = probabilidade estatística; DP = desvio padrão. 
Celina MC, Lacet e Edna Strauss. O virus da hepatite $B$ na doença hepática alcoólica: avaliação clínica $e$ bioquímica. Revista da Sociedade Brasileira de Medicina Tropical 26:201-209, out-dez, 1993.

Tabela 7 - Valores das bilirrubinas: direta total, indireta e total.

\begin{tabular}{lrrrrrr}
\hline & Grupo & N & Média & DP & Min & Max \\
\hline Direta total (mg/\%) & I & 17 & 3,76 & 6,44 & 0,3 & 26,0 \\
& II & 28 & 3,83 & 6,90 & 0,2 & 34,0 \\
& III & 55 & 4,08 & 7,00 & 0,1 & 36,4 \\
Indireta (mg/\%) & I & 17 & 1,54 & 1,61 & 0,2 & 4,8 \\
& II & 28 & 1,80 & 1,98 & 0,0 & 8,6 \\
& III & 55 & 1,45 & 1,99 & 0,1 & 9,7 \\
Total $(\mathrm{mg} / \%)$ & & & & & & \\
& I & 17 & 5,31 & 7,67 & 0,6 & 30,8 \\
& II & 28 & 5,62 & 8,73 & 0,4 & 42,6 \\
& III & 55 & 5,65 & 8,68 & 0,3 & 40,6 \\
\hline
\end{tabular}

"p" =0,316; "p" = probabilidade estatística; DP = desvio padrão.

Tabela 8 - Valores das proteínas plasmáticas nos três grupos de estudo.

\begin{tabular}{lrrrrrr}
\hline & Grupo & N & Média & DP & Min & Max \\
\hline Proteínas totais $(\mathrm{g} / 100 \mathrm{ml})$ & I & 17 & 7,08 & 1,09 & 4,98 & 9,10 \\
& II & 28 & 7,51 & 0,99 & 5,76 & 9,59 \\
& III & 55 & 7,24 & 0,87 & 5,17 & 9,80 \\
Albumina $(\mathrm{g} / 100 \mathrm{ml})$ & I & 17 & 2,84 & 0,78 & 1,20 & 4,23 \\
& II & 28 & 2,93 & 0,54 & 1,94 & 4,01 \\
& III & 55 & 3,10 & 0,68 & 1,80 & 4,94 \\
Alfa 1 (g/100ml) & I & 17 & 0,26 & 0,10 & 0,09 & 0,42 \\
& II & 28 & 0,28 & 0,09 & 0,14 & 0,43 \\
& III & 55 & 0,29 & 0,09 & 0,12 & 0,50 \\
Alfa 2 (g/100ml) & I & 17 & 0,62 & 0,21 & 0,27 & 1,14 \\
& II & 28 & 0,63 & 0,21 & 0,25 & 1,06 \\
Beta $(\mathrm{g} / 100 \mathrm{ml})$ & III & 55 & 0,68 & 0,20 & 0,28 & 1,20 \\
& & & & & & \\
& I & 17 & 0,80 & 0,28 & 0,40 & 1,40 \\
Gama (g/100ml) & II & 28 & 0,79 & 0,43 & 0,00 & 1,67 \\
& III & 55 & 0,94 & 0,34 & 0,00 & 1,68 \\
& I & 17 & 2,56 & 0,78 & 1,60 & 4,72 \\
& II & 28 & 2,89 & 1,31 & 0,92 & 6,67 \\
& III & 55 & 2,19 & 0,87 & 0,65 & 4,16 \\
\hline
\end{tabular}

"p" =0,298; "p" = probabilidade estatística; DP = desvio padrão. 
Celina MC, Lacet e Edna Strauss. O vírus da hepatite B na doença hepática alcoólica: avaliação clínica $e$ bioquínica. Revista da Sociedade Brasileira de Medicina Tropical 26:201-209, out-dez, 1993.

Tabela 9 - Valores dos fatores de coagulação nos três grupos de estudo.

\begin{tabular}{lrrrrrr}
\hline & Grupo & N & Média & DP & Min & Max \\
\hline TAP (\%) & I & 17 & 66,53 & 24,14 & 30 & 100 \\
& II & 28 & 70,57 & 25,46 & 27 & 100 \\
& II & 54 & 73,57 & 20,68 & 28 & 100 \\
Plaquetas $\left(\mathrm{X}^{3} 10 / \mathrm{mm}^{3}\right)$ & I & & & & & \\
& II & 28 & 118,19 & 66,02 & 30 & 255 \\
& III & 48 & 199,63 & 151,31 & 15 & 910 \\
Fator V $(\%)$ & & & & & & \\
& I & 15 & 62,53 & 18,94 & 32 & 95 \\
& II & 24 & 63,42 & 23,36 & 30 & 100 \\
Fibrinogênio $(\mathrm{mg} / \%)$ & III & 40 & 67,50 & 22,62 & 22 & 120 \\
& & & & & & \\
& I & 15 & 307,20 & 107,09 & 191 & 530 \\
& II & 24 & 295,13 & 136,90 & 116 & 625 \\
& III & 38 & 368,58 & 117,43 & 136 & 563 \\
\hline
\end{tabular}

"p" $=0,382 ;$ "p" = probabilidade estatística; $D P=$ desvio padrão.

Tabela 10 - Resultados da aplicação da classificação de Child/Campbell modificada aos três grupos de estudo.

\begin{tabular}{|c|c|c|c|c|c|c|}
\hline \multirow{2}{*}{ Grupo } & \multicolumn{2}{|c|}{ A } & \multicolumn{2}{|c|}{ B } & \multicolumn{2}{|c|}{ C } \\
\hline & $n^{0}$ & $\%$ & $n^{0}$ & $\%$ & $n^{0}$ & $\%$ \\
\hline I & 5 & 29,41 & 8 & 47,06 & 4 & 23,63 \\
\hline II & 10 & 35,71 & 15 & 53,57 & 3 & 10,71 \\
\hline III & 34 & 61,82 & 19 & 34,55 & 2 & 3,64 \\
\hline
\end{tabular}

Análise de contrastes

\begin{tabular}{rccc}
\hline Contraste & GL & $\mathrm{X}^{2}$ & "p" \\
\hline I vs II & 1 & 1,09 & 0,297 \\
I vs III & 1 & 6,96 & 0,008 \\
II vs III & 1 & 2,76 & 0,097 \\
\hline
\end{tabular}

$\mathrm{X}^{2}=$ qui-quadrado; "p" = probabilidade estatística

\section{DISCUSSÃO}

Ao estudarmos pacientes com doença hepática crônica nos quais as etiologias alcoólica e viral se associam, visamos verificar se a somatória das agressões pelo álcool e pelo VHB traduzem ou não doença hepática de maior gravidade.

A prevalência de $42,06 \%$ de infecção pelo VHB encontrada em nossos pacientes com DHA confirma esta associação em nosso meio ${ }^{30}$. Este achado é semelhante ao relatado por vários outros autores $512 \quad 2341$ que encontraram percentuais significativamente mais elevados de AgHBs com ou sem anti-HBc na DHA, embora a determinação do AgHBs, igualmente realizada por outros autores 413 1936 , não tenha detectado índices de positividade aumentada quando comparada com seus grupos controles.

Na população brasileira é observado um aumento crescente da prevalência do VHB à medida que se caminha do Sul para o Leste, Nordeste e Norte do Brasil $^{1018}$, entretanto estes valores são bem inferiores 
Celina MC, Lacet e Edna Strauss. O vírus da hepatite B na doença hepática alcoólica: avaliação clínica $e$ bioquímica. Revista da Sociedade Brasileira de Medicina Tropical 26:201-209, out-dez, 1993.

aos encontrados por nós na DHA. Considerando que nossos pacientes são procedentes de São Paulo, onde a prevalência do VHB é baixa ${ }^{2030}$, este fato nos permite afirmar que o achado de $15,89 \%$ de positividade para o AgHBs na DHA é significativo.

Paradoxalmente, o índice de imunidade de $26,17 \%$ encontrado em nossos pacientes não difere daquele observado em São Paulo ou em outros Estados 27 37. Achamos importante ressaltar que, face a uma positividade para o $\mathrm{AgHBs}$ de $15,89 \%$ seria de se esperar, numa população imunologicamente competente, índices bem mais elevados de anticorpos ${ }^{43}$. Neste sentido nossos resultados divergem daqueles apresentados na literatura ${ }^{5} 23$, onde foram verificadas altas prevalências de anti-HBs, e foi concordante com alguns autores que, como nós, não encontraram aumentos significativos deste anticorpo na $\mathrm{DHA}^{11}$ 1330 .

O resultado das análises comparativas das variáveis relacionadas ao etilismo, em nossa casuística, não foi útil na separação dos grupos à semelhança dos dados de Chevillotte e cols. ${ }^{12}$ que acreditam que a infecção pelo VHB não potencializa a ação do álcool na produção da cirrose, discordando de outros autores ${ }^{4042}$, que analizaram uma população diferente da nossa, ou seja portadores assintomáticos do VHB, referindo tempo de alcoolismo menor nos pacientes AgHBs positivo.

Dados epidemiológicos considerados como fatores de risco ao contágio com o VHB foram analisados, não sendo encontradas evidências epidemiológicos que justificassem a prevalência aumentada de marcadores do VHB na DHA, como referemalguns autores ${ }^{2836}$, diferentemente de outros relatos $^{3} 35$ que valorizam dados relacionados a contatos prévios com o VHB para justificar sua alta prevalência na DHA. Consideramos importante ressaltar que mesmo em casos clinicamente manifestos da hepatite, a fonte de contato para o VHB permanece não determinada em um terço dos $\operatorname{casos}^{8}$.

Parâmetros clínicos e laboratoriais para detectar os marcadores da associação entre DHA e o VHB têm sido utilizados por alguns autores ${ }^{30} 35$ não se encontrando dados que pudessem discriminar os grupos, à semelhança de nossos resultados. Entretanto foi relatado aumento na frequiência de anticorpos do VHB (anti-HBs e anti-HBc) no grupo de cirróticos, com hipertensão portal ${ }^{29}$. Foram também relatados níveis séricos de ALT significativamente mais elevados nos pacientes com alterações histológicas do tipo inflamatórias crônicas não alcoólica, associados a níveis mais elevados de gama GT no grupo com alterações alcoólicas puras ${ }^{5}$ 14. Nesta casuística observamos que, enquanto os níveis médios de AST e GGT estiveram entre 2,3 e 7,5 vezes o valor máximo normal (VMN), os níveis médios de ALT e Fosfatase Alcalina permaneceram entre 1,3 e 2,4 vezes o VMN, condizentes com o esperado durante internação de hepatopatas por alcoolismo.

Disfunção hepatocelular avaliada através de níves séricos de albumina, taxa de bilirrubinas, pseudocolinesterase e tempo de protrombina foram utilizados por alguns autores ${ }^{14} 3040$ sem entretanto determinar parâmetros discriminatórios entre os grupos com marcadores do VHB ou sem contato prévio com o VHB, à semelhança de nossos resultados.

As diferentes variáveis incluídas na classificação clínico-bioquímica de Child/Campbell modificada, quando analisadas separadamente apresentaram pequenas diferenças que não chegaram a ser estatísticamente significantes, todavia quando agrupadas na classificação, a somatória destas pequenas diferenças tornou-se evidente, descriminando o grupo infectado (I) do grupo imune (II) ou sem contato prévio com o VHB (III).

Acreditamos que a associação da DHA com o VHB, acarreta alterações discretas freqüentemente não significantes em termos de atividade clínicobioquímica, entretanto ela é de fundamental importância patogênica no desenvolvimento do hepatocarcinoma ${ }^{7212432333839}$ devendo a vacinação contra o VHB em alcoólatras crônicos ser considerada 15252631 .

\section{SUMMARY}

The aim of our work was to study the prevalence of $H B V$ markers in Alcoholic Liver Disease (ALD) by evaluating clinical and biochemical parameters that could further characterize the association. A prospective and sequential study of 107 patients with ALD was performed, including 83 cases of cirrhosis and 24 cases of alcoholic hepatitis. Dally ingestion of pure ethanol was of at least $70 \mathrm{gm}$ for seven years or more and always associated with hepatocelullar disfunction. According to 
Celina MC, Lacet e Edna Strauss. $O$ vírus da hepatite $B$ na doença hepática alcoólica: avaliação clínica $e$ bioquimica. Revista da Sociedade Brasileira de Medicina Tropical 26:201-209, out-dez, 1993.

the serological profile for $\mathrm{HBV}$ markers the patients were allocated to one of four groups: group $I$ infected (positivity of HBsAg and anti-HBc); group II immunized (positivity of anti-HBs and anti-HBc); group III without $H B V$ markers (negativity of $H B s A g$, anti-HBc and anti$H B s) ;$ group IV isolated anti-HBc. The prevalence of HBSAg positivity in ALD was high: $15.89 \%$ whereas immunity was low: $26.17 \%$ suggesting a great exposure to the virus and a deficient immunological response. No significant statistical differences were found among the three groups when clinical and biochemical parameters were individually considered. Nevertheless, when a Child/Campbell classification was applied, patients with $A L D$ associated with HBV (group I) showed a significant difference, presenting a predominance of child $C$, with a bad prognosis.

Key-words: Alcoholic liver disease (ALD). Hepatitis $B$ virus. AgHBs. Clinical-biochemical evaluation.

\section{REFERÊNCIAS BIBLIOGRÁFICAS}

1. Anderson TW. An introduction to multivariate statistical analysis. John Wiley \& Sons, New York 1952.

2. Antonácio F. Antígeno austrália em doadores de Sangue. Tese de doutorado Universidade de São Paulo, São Paulo, 1971.

3. Attali P, Thibaut N, Buffet C, Briantais MJ, Papoz L, Chaput JC, Etienne JP. Leus marqueurs du virus B chez les alcooliques chroniques. Gastroenterologie clinique et biologique 5:1095-1102, 1981.

4. Basile A, Vitale G, Macor C, Scudeller G. Hepatitis $B$ virus infection in alcoholic cirrhosis. British Medical Journal 282:1705, 1981.

5. Bianchini E, Frosi A, Bertoli G, Bianchi G. Relationships between alcoholism, $\mathrm{HBsAg}$ in serum, liver histology and serum enzymes (CGT, GOT, GPT) in chronic liver diseases: a study of 328 patients. Italian Journal of Gastroenterology 17:14$17,1985$.

6. Bishop YMM, Fienberg SE, Holland PW. Discrete multivariate analysis: theory and practice. Cambrigde, the MIT press, 1975.

7. Brechot C, Nalpas B, Corouce AM, Duhamel G, Callard P, Carnot F, Tiollais P, Berthelot $P$. Evidence that hepatitis $B$ virus has a role in liver-cell carcinoma in alcoholic liver disease. New England Journal of Medicine 306:1384-1387, 1982.

8. Bruguera M. Transmisión de la hepatitis B. Medicina Clinica (Barcelona) 84:312-314, 1985.

9. Campbell DP, Parker DE, Anagnostopoulos CE. survival prediction in portocaval shunts. A computerized statistical analysis. American Journal of Surgery 126:748-751, 1973.

10. Carrilho FJ, Baldy JLS, Takata PK, Adum S, Zeitune JMR. Prevalence and study of assymptomatic carrier of hepatitis B surface antigen in blood donnors in Londrina, South of Brazil. Gastroenterologia Endoscopia digestiva 3:13.-20, 1984.

11. Chalmers DM, Brillen AW, Levi AJ. Evidence for previous hepatitis B virus infection in alcoholic cirrhosis. British Medical Journal 282:819, 1981.

12. Chevillotte G, Durbec JP, Gerolami A, Berthezene $P$, Bidart JM, Camatte $R$. Interaction between hepatitis $B$ virus and alcohol comsuption in liver cirrhosis. An epidemiologic study. Gastroenterology 85:141-145, 1983.

13. Chiaramonte $M$, Heathcote J, Cress $M$, Sherlock S. Detection by three techniques of hepatitis $B$ surface antigen ( $\mathrm{HBsAg}$ ) and determination of $\mathrm{HBsAg}$ and anti-HBs titres in patients with chronic liver disease. Gut 18:1-6, 1977.

14. Coste T, Gié A, Fischer D, Karsenti P, Paraf A. Fréquence des marqueurs du virus $B$ chez les alcooliques chroniques avec ou sans cirrhoses. Gastroenterologie clinique biologique 6:515-516, 1982.

15. Degos F, Brechot C, Nalpas B, Duhamel G, Courouce AM, Tron F, Berthelot P. Hepatitis B vaccination and alcoholic cirrhosis. Lancet $2: 1496$, 1983.

16. Fleiss JL. Statistical methods for rates and proportions. John Wiley \& Sons, New York, 1973.

17. Garcia-Tsao G, Groszmann RJ, Fischer RL, Conn HO, Atterbury CE, Glickman M. Portal pressure presence of gastroesophageal varices and variceal bleeding. Hepatology 5:419-424, 1985.

18. Gayotto LCC, Quarentei AA, Cabral GL. Soroepidemiologia das hepatitis A e B nas regiões dos rios Biá e Alto Juruá, Amazônia Ocidental. Gastroenterologia Endoscopia digestiva 3:106-112, 1984.

19. Gluud C, Gluud B, Aldershvile J, Jacobsen A, Dietrichson O. Prevalence of hepatitis B infection in out-patient alcoholics Infection 12:72-74, 1984.

20. Guimarães RX. Frequência do antígeno australiano em indivíduos normais, índios do Parque Nacional do Xingu e portadores de esquistossomose mansônica. São Paulo, 1973 Tese de doutorado Escola Paulista de Medicina.

21. Hadengue A, N'dri N, Benhamou JP. Relative risk of hepatocellular carcinoma in $\mathrm{HBsAg}$ positive vs alcoholic cirrhosis. A cross-sectional study. Liver 10:147-151, 1990

22. Hislop WS, Follett EAC, Bouchier IAD, Macsween RNM. Serological markers of hepatitis $B$ in patients 
Celina MC, Lacet e Edna Strauss. O vírus da hepatite B na doença hepática alcoólica: avaliação clínica e bioquímica. Revista da Sociedade Brasileira de Medicina Tropical 26:201-209, out-dez, 1993.

with alcoholic liver disease: a multi-centre survey. Journal of Clinical Pathology 34:1017-1019, 1981.

23. Inoue KL, Kojima T, Koyata H, Matsui S, Aoyama $\mathrm{K}$, Konda T, Ishida T, Sasaki H. Hepatitis B virus antigen and antibodies in alcoholic. Etiological role of HBV in liver diseases of alcoholic patients. Liver 5:247-252, 1985.

24. Kobayashi K, Unoura M, Tanaka N, Hattori N. A comparison between hepatocellular carcinoma developing and non-carcinoma-developing patients with cirrhosis over a long follow-up period. Hepatogastroenterology 33:445-448, 1990.

25. Lybecker LA, Mendenhall CL, Marshall LE, Weesner RE, Myre RE. Response to hepatitis B vaccine (HBVac) in the alcoholic. Hepatology 3:807, 1983.

26. Mcmahon BJ, Wainright K, Bulkow L, Parkinson $\mathrm{AJ}$, Lindenbaum $\mathrm{M}$, Wainright $\mathrm{R}$, Helminiak $\mathrm{C}$. Response to hepatitis $\mathrm{B}$ vaccine in Alaska natives with chronic alcoholism compared with non-alcoholic control subjects. American Jounal of Medicine 88:460-464, 1990.

27. Mendes TF, Kutz H, Mexas PPF, Herbert BA. Infection by the hepatitis $B$ virus in patients of a General Hospital. Arquivos de Gastroenterologia 16:73-80, 1979.

28. Mills PR, Follet EA, Urguhart GED, Clements G, Macsween RNM, Watkinson G. Evidence for previous hepatitis B virus infection in alcoholic cirrhosis. British Medical Journal 282:437-438, 1981.

29. Mills PR. Pennington TH, Kay P, Macsween RNM, Watkinson H. Hepatitis Bs antobody in alcoholic cirrhosis. Journal Clinical Pathology 32:778-782, 1979.

30. Mincis M, Guimarães RX, Farinazzo Neto J, Novo NF, Chechter L. Marcadores imunológicos do virus $\mathrm{B}$ da hepatite em alcoolatras e indivíduos normais. Revista Paulista de Medicina 102:205-213, 1984.

31. Nalpas B. Alcool et virus de l'hepatite B: une association insolite aux consequences imprevisibles. Gastroenterologie Clinique biologique 9:385-388, 1985.

32. Nalpas B, Driss F, Pol S, Hamelin B, Hosset C, Brechot C, Berthelot $P$. Association between $\mathrm{HCV}$ and $\mathrm{HBV}$ infection in hepatocellular carcinoma and alcoholic liver disease. Journal of Hepatology 12:7074, 1991.

33. Omata M, Yokosuka O, Imazeki F, Ito Y, Mori J,
Ichiumi K, Okuda K. Correlation of hepatitis B virus DNA and antigens in the liver - a study in chronic liver disease. Gastroenterology 92:192-196, 1987.

34. Pettigrew NM, Goudie RB, Russel RJ, Chaudhurt AKR. Evidence for a role of hepatitis B virus in chronic alcoholic liver disease. Lancet 2:724-725, 1972.

35. Saunders JB, Wodak AD, Morgan-Capner P, White YS, Portmann B, Davis M, Willians R. Importance of markers of hepatitis B virus in alcoholic liver disease. British Medical Joumal 286:1851-1853, 1983.

36. Stigendal L, Hermodsson S, Olsson R. Prevalence of markers of hepatotropic viruses in alcoholic with symptomatic liver cirrhosis or pancreatitis. Scandinavian Journal of Gastroenterology 19:588590,1984

37. Strauss E, Maffei Jr RA, Sá MFG, Dutra AP, Takeda AK. Incidência de $\mathrm{HBsAg}$ e anti-HBs. Frequência do antígeno de superfície da hepatite $B$ e de seus anticorpos detectados por radioimunoensaio, em pessoal hospitalar. Revista do Instituto de Medicina Tropical de São Paulo 25:246-253, 1983.

38. Tamburro $\mathrm{CH}$, Lee HM. Primary hepatic cancer in alcoholic. Clinical Gastroenterology 10:457-477, 1981.

39. Trichopoulos D, Gerety RJ, Sparros L, Tabor E, Xirouchaki E, Munez N, Linsell CA. Hepatitis B and primary hepatocellular carcinoma in a european population. Lancet 2:1217-1219, 1978.

40. Vetter D, Doffoel M, Gut JP, Doffoel S, North ML, Charrault A, Ventre G, Mayer S, Bockel R. Virus de l'hepatite $B$, marqueurs serologiques d'infections virales et immunité humorale au cours de la cirrhose alcoolique. Gastroenterologie clinique et biologique 9:389-395, 1985

41. Villa E, Baldini G, Distalibe S, Pasquinelli C, Antonioli A, Plessi M, Braghiroli D, Barchi T, Monzani A, Manenti F. Alcohol and hepatitis B virus infection. Acta medica scandinavica 703(suppl):97-101, 1985.

42. Villa E, Barchi T, Grisendoi A, Bellentani S, Rubbiani L, Ferreti I, De Palma M, Mamenti F. Susceptibility of chronic symptomless $\mathrm{HBsAg}$ carriers to ethanol-induced hepatic damage. Lancet 2:1243-1244, 1982.

43. Zuckerman AJ. Controversies in immunization against hepatitis B. Hepatology 5:1227-1230, 1985. 